\title{
Prevalence and level of antibodies to the circumsporozoite protein of human malaria parasites in five states of the Amazon region of Brazil
}

\author{
Mercia E Arruda/ ${ }^{+}$, Robert H Zimmerman*, Renata MC Souza, Joseli Oliveira-Ferreira**
}

Departamento de Imunologia, CPqAM-Fiocruz, Av. Moraes Rego s/no-, 50670-420 Recife, PE, Brasil *Florida Medical Entomology Laboratory, University of Florida, Gainesville, FL, US **Departamento de Imunologia, IOC-Fiocruz, Rio de Janeiro, RJ, Brasil

The aim of this study was to determine the prevalence of malaria infection and antibodies against the repetitive epitopes of the circumsporozoite (CS) proteins of Plasmodium falciparum, P. malariae, P. vivax VK210, P. vivax VK247, and P. vivax-like in individuals living in the states of Rondônia, Pará, Mato Grosso, Amazonas, and Acre. Active malaria transmission was occurring in all studied sites, except in Acre. P. falciparum was the predominant species in Pará and Rondônia and P. vivax in Mato Grosso. Infection by P. malariae was low but this Plasmodium species was detected in Rondônia (3.5\%), Mato Grosso (2.5\%), and Pará (0.8\%). High prevalence and levels of serological reactivity against the CS repeat peptides of $\mathrm{P}$. falciparum were detected in Rondônia (93\%) and Pará (85\%). Sera containing antibodies against the CS repeat of P. malariae occurred more frequently in Rondônia (79\%), Pará (76\%), and Amazonas (68\%). Antibodies against the repeat epitope of the standard CS protein of P. vivax VK210, P. vivax VK247, and P. vivax-like were more frequent in Rondônia, Pará, and Mato Grosso. The high frequency of reactions to P. malariae in most of the areas suggests that the infection by this Plasmodium species has been underestimated in Brazil.

Key words: Plasmodium - infection - Amazon region - Brazil

Malaria remains as a serious health problem in the Amazon Basin of Brazil and approximately $99 \%$ of some 600,000 cases notified annually occur in this region (Ministério da Saúde 2003, Coura et al. 2006). Malaria is heterogeneously distributed in the nine states of the Legal Amazon and the number of annual cases fluctuates, increasing in some states where gold mining activities take place, massive migration occurs, and new settlements projects are implemented. However, the states of Pará, Amazonas, and Rondônia are always among the major malaria-endemic regions. Based on microscopic examination from the records of the Brazilian Ministry of Health, $P$. falciparum and $P$. vivax are the most prevalent Plasmodium. The reported prevalence of $P$. malariae is very low $(<1 \%)$. In the last 15 years the number of $P$. vivax has increased and presently accounts for $78 \%$ of all malaria cases reported in the country. Besides the classic $P$. vivax VK210, two species-specific circumsporozoite (CS) protein variants $P$. vivax VK247 (Rosenberg et al. 1989) and P. vivax-like (Qari et al. 1993, 1994) were described. The human $P$. vivax-like parasite has a CS repeat which corresponds to the simian parasite $P$. simiovale. Otherwise the identity of these parasites is not clear, since a line of the human isolates has not yet been obtained and the clinical manifestation of these infections is unknown.

Financial support: OMS/TDR (grant 900550), FNS, CNPq, Fundep/MG, Fiocruz

+Corresponding author: merciaarruda@gmail.com

Received 19 February 2007

Accepted 24 April 2007
Antibodies to sporozoites represent a serological transmission indicator and have been used in epidemiological studies to estimate the level of malaria in endemic areas. It is well known that individuals living in malaria endemic areas exposed to sporozoite infections develop antibodies directly to the repetitive epitopes of the CS protein. The prevalence and levels of anti-sporozoite antibodies to $P$. falciparum increase with age and have been shown to correlate with entomological inoculation rate assessed at the same time and place (Nardin et al. 1979, Druilhe et al. 1986, Del Giudice et al. 1987, Greenwood 1990). In malaria-endemic areas of Brazil it is difficult to appraise entomological data quantitatively and the serological transmission indicator may serve as an alternative in control programs. In these regions very few serological studies to all three human malaria species have been performed (Arruda et al. 1989, Krensmer et al. 1992, Machado et al. 2000). Most studies reported only the prevalence of antibodies to $P$. vivax and its variants. Antibodies to $P$. vivax VK247 sporozoites was first detected in the state of Pará in sera samples from indigenous populations during a survey conducted from 1986 to1988 (Cochrane et al. 1990). A retrospective study showed that the $P$. vivax VK247 and $P$. vivaxlike were present in indigenous populations since 1987 (Arruda et al. 1998). Antibodies to these variants were also detected in other regions of the Brazilian Amazon; in the states of Acre (Kremsner et al. 1992, Branquinho et al. 1993, Marelli et al. 1997, Machado et al. 2000). More recently antibodies to the CS repeats of these two variants were also detected in areas of very low endemicity in the states of Espírito Santo (Arruda, unpublished observations) and São Paulo (Curado et al. 1997).

The aim of the present study was to conduct a seroepidemiological survey of malaria in human populations 
living in endemic areas of the Brazilian Amazon and determine the prevalence of malaria infections and antibody recognition of peptides representing the repetitive epitope of the CS protein of $P$. falciparum, P. malariae, $P$. vivax VK210, P. vivax VK247, and $P$. vivax-like human malaria parasites.

Serological studies may shed light on the dynamics of malaria in area where local health systems are lacking and baseline data are needed to better understand and develop interventions.

\section{MATERIALS AND METHODS}

Study area - Seven randomized surveys were conducted from 1993 to 2003 in five states of the Amazon region of Brazil (Fig. 1). A total of 1206 serum samples and blood films were collected from different populations in the states of Pará, Rondônia, Mato Grosso, Acre, and Amazonas. All participants were interviewed directly or through a respondent inhabitant and allocated a unique identification number. Informed consent was obtained from all individuals before admission into the study. The information collected included name, sex, age, and time remained in the area.

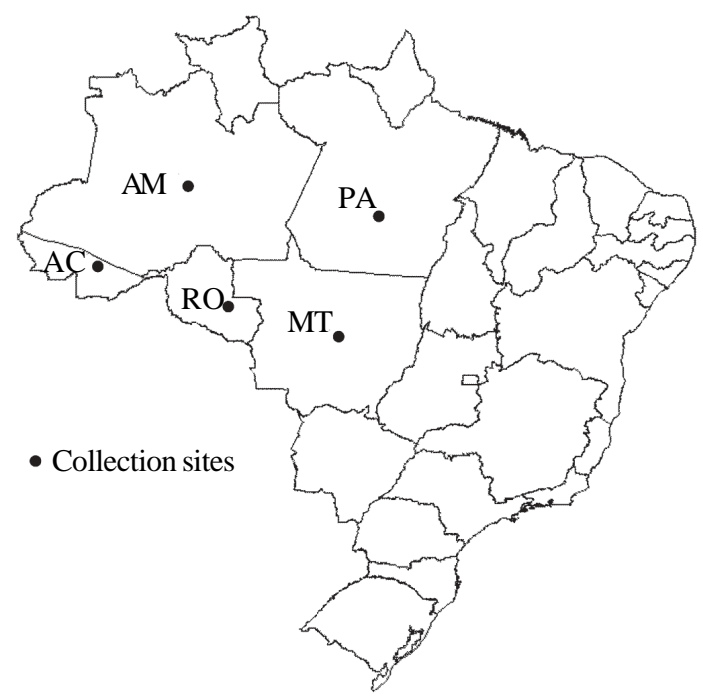

Fig. 1: location of the collection sites. AC, Acre; AM, Amazonas; MT, Mato Grosso; PA, Pará; RO, Rondônia.

Results discussed in this article are related to samples of blood randomized collected during the development of the following projects: "A longitudinal study to determine whether there is seasonal fluctuation in the incidence of Plasmodium falciparum, P. vivax and $P$. malariae and in the species of mosquitoes responsible for their transmission in the State of Pará, Amazon region of Brazil", "Identificação dos anofelinos responsáveis pela transmissão das diferentes espécies de malárias existentes nas áreas de garimpo dos Municípios de Peixoto de Azevedo e Matupá, Estado de Mato Grosso" and "Revisitando a Amazônia de Carlos Chagas: da borracha à biodiversidade". The ethics committee of the Fundação Oswaldo Cruz approved the first two projects.
The first survey was conducted in 1993 in Pará, where 119 serum samples and blood films were taken from natives living in four locations (Asa da Arara and Apinajes in the municipality of São João do Araguaia, and Veadas and Televisão in the municipality of Itupiranga). These locations were chosen based on a previous survey conducted during 1983-1985 that showed year-round transmission of P. vivax, P. falciparum and P. malariae (Arruda et al. 1986). In 1994 and 1995, serum samples and blood films were collected in Mato Grosso from 471 gold miners in 14 different mining sites in the municipalities of Peixoto de Azevedo and Matupá. In 1995, a total of 142 serum samples and blood films were collected in Rondônia from farmers living in Machadinho, who had migrated from southern Brazil to colonize this part of the Amazon in mid-1980. In 1997 a total of 180 serum samples and blood films were collected from stable riverine populations living along the Acre River. In the same survey 170 serum samples and blood films were also collected in the state of Amazonas along the Purus River, from native descendents of families that worked in the rubber plantations at the beginning of last century. In 2002, in the same state, 124 serum samples and blood films were collected from individuals that over the last several years invaded the suburban areas of the capital Manaus. The populations tested during the surveys were representative of the major malaria-endemic areas of the Amazon region of Brazil.

Blood collection - Approximately $5 \mathrm{ml}$ of venous blood was collected from consenting individuals and allowed to clot for 5-6 hours at room temperature. Serum samples were stored in $-20^{\circ} \mathrm{C}$. Thick and thin blood films were prepared soon after the blood samples were drawn, stained with Giemsa and examined under oil immersion (x 100) with a conventional light microscope. A minimum of 250 fields was examined before a slide was declared negative. The parasitological examination of the blood slides was done in the local areas and individuals found positive for malaria parasites were treated.

ELISA test for the detection of anti-CS antibodies The ELISA for detection of antibodies to the CS protein was conducted as described by Wirtz et al. (1990). All serum samples were tested in duplicate at a dilution of $1: 100$. Synthetic peptides used as antigen were the repeats of the CS proteins of $P$. vivax VK210 (DGQ PAGDRAA-(GQPAGDRAD)2-GQPAG, $P$. viva $x$ VK247 (ANGAGNQPG)3-ANGAGN, $P$. vivax-like (APGAN QEGGAA)3, $P$. falciparum (NANP)8, and $P$. malariae (GNAA)2-GNDA-(GNAA)5. The mouse monoclonal antibodies Pf2A10, PvNSV3, Pv247, and Pm were used as positive controls for Pf, PVK210, PVK247, and Pm synthetic peptides. Polyclonal serum used as positive control for $P$. vivax-like was obtained from a wild monkey infected with $P$. simium and reacted strongly against the synthetic peptide. Since a level of antibody equal to 1:40 or less may be questionable as an indication of past or present malaria in endemic areas we elected to consider positive only sera with ELISA endpoints of 1:100 or higher. We calculated the cutoff value for each peptide using twenty serum samples from healthy malaria naive control individuals who never had a past 
history of malaria and who had never been visited malaria transmission areas. Samples were considered positive when the mean OD values were above the mean plus 3 standard deviations of healthy control group.

\section{RESULTS}

Blood film results demonstrated that malaria transmission was occurring in all populations studied, except in Acre (Table). The level of parasitemia was low in most individuals, usually lower than $0.02 \%$. Mature asexual parasites were detected in only a few smears and three individuals showed mature gametocytes in the peripheral blood. Thick and thin blood films evaluation showed that the prevalence of malaria infection was significantly higher in Pará (40\%) followed by Rondônia (17.6\%) and Mato Grosso (15\%). P. falciparum was more prevalent in Pará (29\%) and Rondônia (9.5\%) and P. vivax in Pará $(10.1 \%)$ and Mato Grosso (7.6\%). Infection by P. malariae was low but detected in Rondônia (3.5\%), Mato Grosso (2.5\%), and Pará $(0.8 \%)$. The overall prevalence of malaria infection was highest in Pará followed by Rondônia and Mato Grosso (Table).

The prevalence of antibodies to the CS repeats of $P$. falciparum, $P$. malariae, and $P$. vivax was greater in migrants from Rondônia and natives from Pará compared to the other populations surveyed (Fig. 2). In Mato Grosso the prevalence of antibodies to all the CS repeats was lower but the frequency distribution of positive to all CS repeats was similar to Rondônia and Pará. In Amazonas the prevalence of antibodies was higher for the CS repeats of $P$. falciparum and P. malariae although infection by $P$. malariae was not detected by thick and thin blood smear examination. The high prevalence of antibodies to $P$. malariae contrasting with low prevalence to $P$. vivax suggests that $P$. malariae could be misdiagnosed as $P$. vivax in this area. In Acre plasmodial infection was not detected and the prevalence of antibodies to all three CS repeats was very low. This may reflect a low level of exposure of the stable riverine populations to Plasmodium-infected Anopheles mosquitoes.

Antibodies against the repeat epitope of the CS protein of $P$. vivax VK210, the variant VK247 and P. vivaxlike were detected in all of the states surveyed. High levels of anti-sporozoite antibodies against $P$. vivax VK210 and P. vivax VK247 were detected, especially in migrants from Rondônia and natives from Pará while P. vivax-like was more prevalent in Rondônia. In
Amazonas and Acre the prevalence of antibodies to the CS repeats of $P$. vivax VK210 and both variants was very low.

The geometric mean titer (GMT) of antibodies to the CS repeats varied quite appreciated among the studied sites (Fig. 3). In Rondônia the GMT were higher for all CS repeats when compared to the other states. In Pará the highest GMT was to the CS repeat of $P$. vivax VK210 followed by P. falciparum. In Mato Grosso, Amazonas, and Acre the GMT for all species was low.

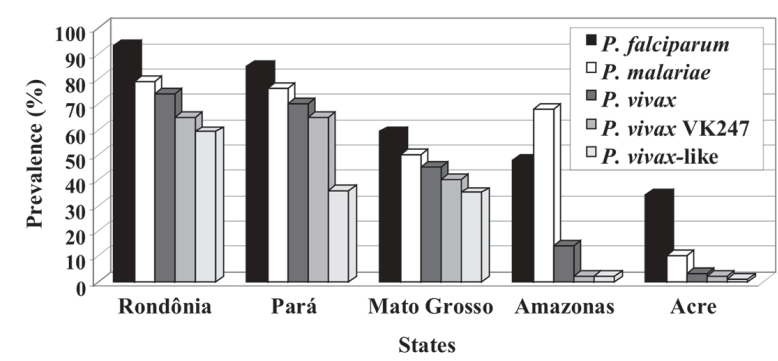

Fig. 2: prevalence of antibodies to the circumsporozoite repeats of Plasmodium falcipa-rum (Pf), P. malariae $(\mathrm{Pm})$, P. vivax $(\mathrm{Pv})$, P. vivax VK247 (Pv247), and P. vivax-like (Pv-like) in endemic areas of the Amazon Region of Brazil. Differences in proportion were evaluated by Chi square test. Rondônia: Pf vs Pm (Chi = 9.236 p = 0.002), Pf vs Pv (Chi = 15.669 p $<0.0001)$, Pf vs PvK247 (Chi = 30.147 p <0.0001), Pf vs Pv-like (Chi = 41.880 p < 0.0001); Pará: $\operatorname{Pf}$ vs $\mathrm{Pm}(\mathrm{Chi}=5.537 \mathrm{p}<0.018)$, Pf vs Pv $(\mathrm{Chi}=$ $6.923 \mathrm{p}<0.008)$, Pf vs Pv247 (Chi $=11.789 \mathrm{p}<0.0006)$, Pf vs Pv-like (Chi $=57.126 \mathrm{p}<0.0001)$; Acre: Pv247 vs Pm $(\mathrm{Chi}=51.002 \mathrm{p}<0.0001), \operatorname{Pv} 247$ vs $\mathrm{Pv}$-like $(\mathrm{Chi}=10.418 \mathrm{p}<0.0012)$.

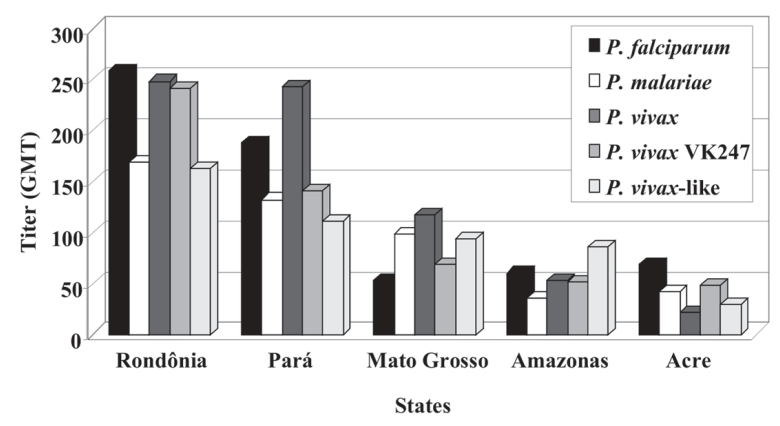

Fig. 3: geometric mean titer (GMT) of antibodies to the circumsporozoite repeats of Plasmodium falciparum, P. malariae, P. vivax, P. vivax VK247, and $P$. vivax-like in endemic areas of the Amazon region of Brazil.

TABLE

Frequency of patent malaria infections as determined by blood smears collected in five states of the Amazon region of Brazil

\begin{tabular}{lccrr}
\hline State & $\begin{array}{c}\text { No. tested } \\
\text { \% positive })\end{array}$ & $\begin{array}{c}\text { Plasmodium falciparum } \\
(\mathrm{Pf})\end{array}$ & $\begin{array}{c}\text { P. malariae } \\
(\mathrm{Pm})\end{array}$ & $\begin{array}{c}\text { P. vivax } \\
(\mathrm{Pv})\end{array}$ \\
\hline Rondônia & $142(17.6)$ & $13(9.2)$ & $5(3.5)$ & $7(4.9)$ \\
Mato Grosso & $471(15.1)$ & $23(4.9)$ & $12(2.6)$ & $36(7.6)$ \\
Pará & $119(40.3)$ & $35(29.4)$ & $1(0.8)$ & $12(10.1)$ \\
Acre & $180(0.0)$ & $0(0.0)$ & $0(0.0)$ & $0(0.0)$ \\
Amazonas & $294(7.1)$ & $12(4.1)$ & $0(0.0)$ & $9(3.1)$ \\
Total & $1206(13.7)$ & $83(6.8)$ & $18(1.5)$ & $64(5.3)$ \\
\hline
\end{tabular}

Differences in proportion were evaluated by Chi square test. Mato Grosso: Pf vs Pv (Chi=4.176 p = 0.041), Pv vs Pm (Chi = 16.648 p < 0.0001); Pará: Pf vs Pm $(\mathrm{Chi}=48.400$ p < 0.0001), Pf vs Pv $(\mathrm{Chi}=20.870 \mathrm{p}<0.0001)$, Pv vs Pm $($ Chi $=0.012 \mathrm{p}=6.193)$. 


\section{DISCUSSION}

In the Brazilian Amazon despite the high prevalence of human malaria parasites there are still states where little information about the distribution of cases caused by $P$. vivax, $P$. falciparum, and $P$. malariae is available.

Data for this seroprevalence study was collected in the course of seven random surveys conducted from 1993 to 2003 in order to evaluate the Plasmodium species and determine the prevalence of malaria infection and antibody recognition of peptides representing the repetitive epitope of the CS protein of $P$. falciparum, $P$. malariae, $P$. vivax VK210, $P$. vivax VK247, and $P$. vivax-like human malaria parasites. The individuals included in these surveys were living in the states of Rondônia, Pará, Mato Grosso, Amazonas, and Acre.

Blood smear results demonstrated that active malaria transmission was occurring in all studied sites, except in Acre. The level of parasitemia was low in most individuals, usually lower than $0.02 \%$. Mature asexual parasites were detected in only few smears and three individuals showed mature gametocytes in the peripheral blood. There was a high prevalence of antibodies to the CS repeat of $P$. malariae in the states of Rondônia, Pará, and Amazonas. The high prevalence of antibodies against $P$. malariae shows the discrepancy between our data and the official cases reported by SIVEP suggesting that this Plasmodium species seems to be more common than suspected. The failure to report $P$. malariae in endemic areas is not surprising since the only official method for malaria diagnosis in Brazil is the microscopic examination of thick blood smear stained with Giemsa. In this procedure it is not possible to assess the morphology of infected red blood cells and the parasite-altered shape can lead to a mistaken identification of $P$. malariae as $P$. vivax. However, the antibody response to $P$. malariae antigens suggests that this parasite could possible be more prevalent than $P$. vivax in human populations in Rondônia, Amazonas, and Pará. Recently, Cavasini et al. (2000) and Scopel et al. (2004) found significantly more cases of $P$. malariae infection in patients from Rondônia and Mato Grosso, when using nested polymerase chain reaction and comparing the results of Plasmodium species with conventional on-site microscopy of Giemsa-stained thick smears. These results point to the need for the development or use of a more accurate diagnosis method to distinguish between $P$. malariae and $P$. vivax. This is particularly important in view of the fact that the drug of choice for antimalarial therapy depends on the parasite species (Scopel et al. 2004).

The number of individuals with antibodies to the CS repeats of $P$. falciparum, P. malariae, and $P$. vivax were higher in migrants from Rondônia and natives from Pará, which coincided with the total of cases, reported by the Ministry of Health during the year of our survey (Rondônia 133,246 reported cases in 1995 and Pará 135,952 reported cases in 1993). However, this number could increase since repeated inoculations are needed to get a detectable level of antibodies (Mota et al. 1996). Wirtz et al. (1990) have shown that only 19\% of subjects with confirmed acute $P$. vivax infection had detectable antibodies against CS protein. The age of indi- viduals with detectable anti-sporozoite antibodies ranged from less than 5 to 64 years old.

In Mato Grosso, the prevalence of antibodies to the CS repeats of P. falciparum and P. vivax was 59 and $45 \%$, respectively. In the surveyed area there is a continuous influx of new miners who never had malaria and others that returned after a few years from malaria free areas. This may account for the moderate prevalence in Mato Grosso when compared to Rondônia and Pará.

Antibodies against the CS protein of $P$. vivax VK247 variant occurred more frequently in Rondônia, Pará, and Mato Grosso and to a lesser extend against the repeat $P$. vivax-like. In Amazonas the prevalence of antibodies to the CS repeat of both variants was very low. This might reflect the recent introduction of these variants in the area or differences in the development of these variants in the local vector, resulting in a lower transmission rate.

The lowest prevalence of antibodies and patent infections for all Plasmodium species at the study sites were detected in the riverine populations of Acre. According to Funasa the number of reported cases during the year of our survey was low (6070 cases). One possible explanation for the low prevalence and titers may be related to the history and stability of these populations and the vectors species. Indeed, the participants of our study live in small-scattered communities (2-20 families) along the rivers. They practice subsistence agriculture, there is a low level of migration between communities and malaria is unstable with only occasional outbreaks. In addition, vector abundance was low, particularly for the principal vector in the Amazon Region, An. darlingi (Zimmerman et al. 1999) when compared to the other sites studied (Rondônia, Lourenço et al. 1989, Pará, de Arruda et al. 1986). In contrast, in Machadinho, Rondônia state, An. darlingi was present in high numbers and represented $98.3 \%$ of the anophelines collected (Lourenço et al. 1989).

GMT were calculated to compare differences in antibody levels between the study groups. In general, the ELISA GMT titers for $P$. vivax in Pará were considerably lower than those obtained from the examination of samples from Indians belonging to the A-Ukre and Pucanuv tribes, living in this same region of the Amazon. The overall GMT for the $P$. vivax VK247 variant in the Indians tribes living in Pará was 1073 (Arruda et al. 1998), while in natives of the same state reached only 353. The GMT to the CS repeat sequence of $P$. vivax VK210 and the variant VK247 showed similar trends in Rondônia and Pará while $P$. vivax-like were almost twice as prevalent in Rondônia.

The results of this study demonstrate that $P$. malariae infection is widely distributed in the Amazon Region of Brazil and it is more common than previously recognized. The transmission of the two variant strains of $P$. vivax VK247 and $P$. vivax-like is a common phenomenon in this region and vary from state to state even in geographically small areas.

In conclusion, seroepidemiological stratification of malaria showed to be a valuable method to recognize how variations in habits and years of contact with vectors contribute to the level of antibodies and intensity of transmission in malaria-endemic areas. 


\section{ACKNOWLEDGEMENTS}

To Dr Ruth Nussensweig and Elisabeth Nardin, from the New York University, for allowing us to use their facilities. To Filipe Dantas-Torres for his critical and English review of the manuscript. To the staff of the Fundação Nacional de Saúde (Ministério da Saúde) for their technical assistance and logistic support. Also to the Ministério da Defesa and Secretaria Estadual de Saúde do Acre for logistic support.

\section{REFERENCES}

Arruda M, Carvalho BC, Nussenzweig RS, Maracic M, Ferreira AW, Cochrane AH 1986. Potential vectors of malaria and their different susceptibility to Plasmodium falciparum and $P$. vivax in Northern Brazil identified by immunoassay. Am J Trop Med Hyg 35: 873-881.

Arruda M, Nardin EH, Nussenzweig RS, Cochrane AH 1989. Sero-epidemiological studies of malaria in Indian tribes and monkeys of the Amazon Basin of Brazil. Am J Trop Med Hyg 41: 379-385.

Arruda ME, Souza RC, Veiga ME, Ferreira AF, Zimmerman RH 1998. Prevalence of Plasmodium vivax variants VK247 and P. vivax-like human malaria: a retrospective study in indigenous Indian populations of the Amazon region of Brazil. Trans $R$ Soc Trop Med Hyg 92: 628-628.

Branquinho MS, Lagos CB, Rocha RM, Natal D, Barata JM, Cochrane AH, Nardin E, Nussenzweig RS, Kloetzel JKl 1993. Anophelines in the State of Acre, Brazil, infected with Plasmodium falciparum, $P$. vivax, the variant $P$. vivax VK247 and P. malariae. Trans R Soc Trop Med Hyg 87: 391-394.

Cavasini MTV, Ribeiro WL, Kawamoto F, Ferreira MU 2000. How prevalent is Plasmodium malariae in Rondonia Western Brazilian Amazon? Rev Soc Bras Trop Med 33: 489-492.

Cochrane AH, Nardin EH, de Arruda M, Maracic M, Clavijo P, Collins WE, Nussenzweig RS 1990. Widespread reactivity of human sera with a variant repeat of the circumsporozoite protein of Plasmodium vivax. Am J Trop Med Hyg 43: 446-451.

Coura JR, Suarez-Mutis M, Ladeia-Andrade S 2006. A new challenge for malaria control in Brazil: asymptomatic Plasmodium infection-a review. Mem Inst Oswaldo Cruz, 101: 229-237.

Curado I, Duarte AM, Lal AA, Oliveira SG, Kloetzel JK 1997. Antibodies anti bloodstream and circumsporozoite antigens (Plasmodium vivax and Plasmodium malariae/P. brasilianum) in areas of very low malaria endemicity in Brazil. Mem Inst Oswaldo Cruz 92: 235-243.

Druilhe P, Marc JP, Miltgen F, Mazier D, Parent G 1986. Levels of antibodies to Plasmodium falciparum sporozoite surface antigens reflect malaria transmission rates and are persistent in the absence of reinfection. Infect Immun 53: 393-397.

Del Giudice G, Engers HD, Tougne C, Biro SS, Weiss N, Verdini AS, Pessi A, Degremont AA, Freyvogel TA, Lambert PH 1987. Antibodies to the repetitive epitope of Plasmodium falciparum circumsporozoite protein in Tanzanian community: a longitudinal study of 132 children. Am J Trop Med Hyg 36: 203-212.

Greenwood BM 1990. Immune responses to sporozoite anti- gens and their relationship to naturally acquired immunity to malaria. Bull WHO 68: 184-190.

Kremsner PG, Neifer S, Bienzle U, Rocha RM, Maracic M, Clavijo P, Nussenzweig RS, Cochrane AH 1992. Prevalence and level of antibodies to the circumsporozoite proteins of human malaria parasites, including a variant of Plasmodium vivax, in the population of two epidemiologically distinct areas in the state of Acre, Brazil. Trans R Soc Trop Med Hyg 86: 23-27.

Lourenço-de-Oliveira R, Guimarães AE, Arlé M, Silva TF, Castro MG, Motta MA, Deane LM 1989. Anopheline species: some of their habits and relation to malaria in endemic areas of the state of Rondônia, Amazon Region of Brazil. Mem Inst Oswaldo Cruz 84: 501-514.

Machado RLD, Povoa M 2000. Distribution of Plasmodium vivax variants (VK210, VK247 and $P$. vivax-like) in three endemic areas of the Amazon region of Brazil and their correlation with cloroquine treatment. Trans R Soc Trop Med Hyg 94: 177-181.

Marelli MT, Branquinho MS, Hoffman EHE, Benevento CM, Natal D, Taipe-Lagos CB, Kloetzel JK 1997. ELISA with $P$. vivax-like/P. semiovale CS repeats in human sera and anophelines from the state of Acre, Brazil. Mem Inst Oswaldo Cruz 92: 268.

Ministério da Saúde 2003. Secretaria de Vigilância em Saúde, Programa Nacional de Prevenção e Controle da Malária, Brasília, DF.

Mota J, Coreno O, Cochrane AH, Ramos C 1996. Prevalence and antibodies to the repeat epitope of the circumsporozoite protein of Plasmodium vivax in San Luis Potosi, Mexico. Arch Med Res 27: 233-236.

Nardin EH, Nussenweig RS, McGragor IA, Bryan JH 1979. Antibodies to sporozoites: their frequent occurrence in individual living area of hyperendemic malaria. Science 206: 597-599.

Qari SH, Collins WE, Lobel HO, Taylor F, Lal AA 1994. A study of polymorphism in the circumsporozoite protein of human malaria parasites. Am J Trop Med Hyg 50: 45-51.

Qari SH, Shi Y-P, Goldman V, Udhayakumar MPAlpers, Collins WE, Lal AA 1993. Identification of Plasmodium vivax-like human malaria parasite. Lancet 341: 780-783.

Rosenberg, R, Wirtz, RA, Lanar DE, Sattabongkot J, Hall T, Watters AP, Prasittisuk C 1989. Circumsporozoite protein heterogeneity in the human malaria parasite Plasmodium vivax. Science 245: 973-976.

Scopel KKG, Fontes CJF, Nunes AC, Horta MF, Braga EM 2004. High prevalence of Plasmodium malariae infections in a Brazilian Amazon endemic area (Apiacas - Mato Grosso) as detected by polymerase chain reaction. Acta Trop 90: 61-64.

Wirtz RA, Rosenberg R, Sattabongkot J, Webster HK 1990. Prevalence of antibody to heterologous circumsporozoite protein of Plasmodium vivax in Thailand. Lancet 336: 593-595.

Zimmerman, RH, Paulino FX, de Arruda, M 1999. A survey of the Anopheles mosquitoes (Diptera: Culicidae) along the Acre and Purus Rivers, Amazon basin, Brazil. Acta Amazônica 29: 165-171. 
